\title{
The EvtGen-Based Model for the Monte-Carlo Generation of the Rare Radiative Leptonic B-decays
}

\author{
Alexander Popov* \\ Lomonosov Moscow State University Skobeltsyn Institute of Nuclear Physics \\ E-mail: av.popovephysics.msu.ru

\section{Nikolai Nikitin} \\ Lomonosov Moscow State University Skobeltsyn Institute of Nuclear Physics \\ E-mail: nnikit@mail.cern.ch

\section{Daria Savrina} \\ Lomonosov Moscow State University Skobeltsyn Institute of Nuclear Physics \\ E-mail: Daria.Savrina@cern.ch
}

Here we present the brief description of the decay model for the simulation of rare radiative leptonic decays $\left(\bar{B}_{d, s}^{0}, B_{d, s}^{0}\right) \rightarrow \gamma \ell^{+} \ell^{-}$in the framework of the EvtGen package adopted for the LHCb software.

The XIXth International Workshop on High Energy Physics and Quantum Field Theory, QFTHEP2010 September 08-15, 2010

Golitsyno, Moscow, Russia

\footnotetext{
* Speaker.
} 


\section{Introduction}

Rare radiative leptonic decays $B_{q} \rightarrow \gamma \ell^{+} \ell^{-}$are produced by $b \rightarrow q$ (where $q=\{d, s\}$ ) quarks transitions (so-called Flavor Changing Neutral Currents). These currents are forbidden at a tree level in the framework of the Standard Model (SM) and occur at the lowest order of the perturbation theory only through one-loop "penguin" and "box" diagrams. The "box" diagram contribution is suppressed relative to the "penguin" approximately by a factor $\left(m_{t} / M_{W}\right)^{2} \approx 5$. The branching ratios of these decays in the SM are very small: $\sim 10^{-8}$ for decays of the $B_{s}$-mesons and $\sim 10^{-10}$ for the decays of the $B_{d}$-mesons [1] - [3]. This fact gives us an opportunity to check the SM predictions in a high perturbative order and to search for the "new physics" above the suppressed SM signal.

The correct Monte-Carlo simulation is of great importrance for the search of the rare radiative leptonic $B$-decays at LHC. The purpose of the current paper is to introduce the new EvtGen model BSTOGLLMNT intended for this simulation. It includes the theoretical features - such as the contribution of the weak annihilation, the dependence of the Wilson coefficients on the scale parameter in the NLO approach, resonant contributions - which may be useful for correct simulation of the mentioned decays for LHCb.

\section{Theoretical overview}

The $b \rightarrow q$ transitions with $C P$-violation effects are convenient to describe in terms of the effective Hamiltonian $H_{\mathrm{eff}}^{b \rightarrow q}$ in the Wilson expansion form [4, 5, 6]:

$$
\begin{aligned}
& H_{\mathrm{eff}}^{b \rightarrow q}=\frac{G_{\mathrm{F}}}{\sqrt{2}} V_{t b} V_{t q}^{*}\left[\left(1+\lambda_{u}^{(q)}\right)\left(C_{1}(\mu) O_{1}^{(c)}(\mu)+C_{2}(\mu) O_{2}^{(c)}(\mu)\right)-\right. \\
& \left.-\lambda_{u}^{(q)}\left(C_{1}(\mu) O_{1}^{(u)}(\mu)+C_{2}(\mu) O_{2}^{(u)}(\mu)\right)+\sum_{i=3} C_{i}(\mu) O_{i}(\mu)\right]+(\bar{b} \rightarrow \bar{q}),
\end{aligned}
$$

where $G_{\mathrm{F}}$ is the Fermi constant, $V_{q_{1} q_{2}}$ are the Cabibbo-Kobayashi-Maskava matrix elements, $\lambda_{u}^{(q)}=$ $V_{u b} V_{u q}^{*} / V_{t b} V_{t q}^{*}$. In (1.1) $C_{i}(\mu)$ form a set of the Wilson coefficients. The value of the scale parameter $\mu$ is of the order of a $b$-quark mass here. It separates the hard and soft contributions of the strong interaction. The soft contribution is contained in the matrix elements of the basic operators $O_{i}(\mu)$ between the initial and the final hadronic states. We should note that the accuracy of the Wilson coefficients in the next to the leading logarithms order approximation (NLO) does not exceed the $15 \%$ level as well as the accuracy of the calculations of the hadronic matrix elements. So, the NLO approximation is quite enough for the numerical calculations of the matrix elements.

Either $C_{9 V}^{\mathrm{eff}}(q)(\mu, s)$ (in case of the $b \rightarrow q$ transition, $s$ is the square of the invariant dilepton mass) or $C_{9 V}^{\text {eff }(\bar{q})}(\mu, s)$ (in a charge conjugated case) Wilson coefficient contains the contributions from the $u \bar{u}$ - and $c \bar{c}$-pairs and $\rho^{0}-, \omega-, J / \psi-, \psi^{\prime}-, \psi(3770)-, \psi(4040)-, \psi(4160)-$ and $\psi(4415)-$ resonances as well as $C P$-violation effects [ $\left[\begin{array}{l}\text {. } \\ \text {. }\end{array}\right.$. The $s$-dependence of the resonance contribution is modified according to [7].

One may write the following equations for the effective coefficients:

$$
\begin{aligned}
& C_{9 V}^{\operatorname{eff}(q)}(\mu, s)=C_{9 V}(\mu)+C_{\text {res }}^{(1)}(\mu, s)+\lambda_{u}^{(q)} C_{\text {res }}^{(2)}(\mu, s), \\
& C_{9 V}^{\text {eff }(\bar{q})}(\mu, s)=C_{9 V}^{*}(\mu)+C_{\text {res }}^{(1)}(\mu, s)+\lambda_{u}^{(q) *} C_{\text {res }}^{(2)}(\mu, s) .
\end{aligned}
$$


The $C_{9 V}(\mu), C_{\mathrm{res}}^{(1)}(\mu, s)$ and $C_{9 V}^{*}(\mu)$ coefficients are defined in [3].

Below the definition of the form-factors for the $\bar{B}_{q}^{0} \rightarrow \gamma$ transitions (where $q=\{d, s\}$ ) is presented ${ }^{1}$. The following kinematical notations are used for it: $p^{\mu}$ is the 4-momentum of the $B_{q^{-}}^{0}$ or $\bar{B}_{q}^{0}$-meson, $k^{\mu}$ is the 4-momentum of the photon, $k_{1}^{\mu}$ is the 4-momentum of the positively charged lepton $\ell^{+}$and $k_{2}^{\mu}$ is the 4-momentum of the negatively charged lepton $\ell^{-}$. These 4-momenta satisfy the following equations:

$$
p=k+k_{1}+k_{2}=k+q ; \quad p^{2}=M_{1}^{2} ; \quad q^{2}=s ; \quad k^{2}=0 ; \quad k_{1}^{2}=k_{2}^{2}=m^{2} .
$$

The effective tensor and pseudotensor form-factors take into account the $C P$-violation effects. With these notations the form-factors of the $\bar{B}_{q}^{0} \rightarrow \gamma$ transition may be defined in the following way [1, 2]:

$$
\begin{aligned}
\left\langle\gamma(k, \varepsilon)\left|\bar{q} \gamma_{\mu} \gamma_{5} b\right| \bar{B}_{q}^{0}\left(p, M_{1}\right)\right\rangle & =i e \varepsilon_{\alpha}^{*}\left(g_{\mu \alpha}(p k)-p_{\alpha} k_{\mu}\right) \frac{F_{A}\left(q^{2}\right)}{M_{1}}, \\
\left\langle\gamma(k, \varepsilon)\left|\bar{q} \gamma_{\mu} b\right| \bar{B}_{q}^{0}\left(p, M_{1}\right)\right\rangle & =e \varepsilon_{\alpha}^{*} \varepsilon_{\mu \alpha \xi \eta} p_{\xi} k_{\eta} \frac{F_{V}\left(q^{2}\right)}{M_{1}} \\
\left\langle\gamma(k, \varepsilon)\left|\bar{q} \sigma_{\mu \nu} \gamma_{5} b\right| \bar{B}_{q}^{0}\left(p, M_{1}\right)\right\rangle(p-k)_{v} & =e \varepsilon_{\alpha}^{*}\left(g_{\mu \alpha}(p k)-p_{\alpha} k_{\mu}\right) F_{T A}\left(q^{2}, 0\right), \\
\left\langle\gamma(k, \varepsilon)\left|\bar{q} \sigma_{\mu \nu} b\right| \bar{B}_{q}^{0}\left(p, M_{1}\right)\right\rangle(p-k)_{v} & =i e \varepsilon_{\alpha}^{*} \varepsilon_{\mu \alpha \xi \eta} p_{\xi} k_{\eta} F_{T V}\left(q^{2}, 0\right) .
\end{aligned}
$$

where tensor $\left(F_{T V}\right)$ and pseudotensor $\left(F_{T A}\right)$ form-factors depend on the 4-momenta $k$ of a real $\left(k^{2}=0\right)$ and $q=p-k-$ virtual $\left(q^{2}=s\right)$ photons. The latter one then converted to the $\ell^{+} \ell^{-}$-pair. The first argument of the $F_{T V T A}\left(q_{1}^{2}, q_{2}^{2}\right)$ functions is the square momentum of the (real or virtual) photon, which is emitted due to the effective vertex. This vertex is described with the Hamiltonian of the $b(\bar{b}) \rightarrow q(\bar{q})\left(\ell^{+} \ell^{-}, \gamma\right)$ transitions.

The tensor and pseudotensor form-factors in (1.3) correspond to the case when the real photon is emitted by the valent quarks. Taking into account the emission of the virtual photon by the valent quarks and the weak annihilation contribution one finds the following explicit view of these form-factors:

$$
\begin{aligned}
& F_{T V}^{b \rightarrow q}\left(q^{2}\right)=\left(1+\frac{m_{q}}{m_{b}}\right)\left(F_{T V}\left(q^{2}, 0\right)+F_{T V}\left(0, q^{2}\right)\right)-\frac{16}{3}\left(\lambda_{u}^{(q)}+\lambda_{c}^{(q)}\right) \frac{a_{1}}{C_{7 \gamma}} \frac{f_{B_{q}}}{m_{b}}, \\
& F_{T A}^{b \rightarrow q}\left(q^{2}\right)=\left(1-\frac{m_{q}}{m_{b}}\right)\left(F_{T A}\left(q^{2}, 0\right)+F_{T A}\left(0, q^{2}\right)\right),
\end{aligned}
$$

where $a_{1}=C_{1}+C_{2} / 3$ and $f_{B_{q}}$ is the real positive lepton constant of the $\bar{B}_{q}^{0}$-meson decay, which is defined by the matrix element value: $\left\langle 0\left|\bar{q} \gamma^{\mu} \gamma^{5} b\right| \bar{B}_{q}^{0}\left(p, M_{1}\right)\right\rangle=i f_{B_{q}} p^{\mu}$.

The form-factors for the $B_{q}^{0} \rightarrow \gamma$ transition are expressed through the $\bar{B}_{q}^{0} \rightarrow \gamma$ transitions:

$$
F_{T V}^{\bar{b} \rightarrow \bar{q}}\left(q^{2}\right)=\left(1+\frac{m_{q}}{m_{b}}\right)\left(F_{T V}\left(q^{2}, 0\right)+F_{T V}\left(0, q^{2}\right)\right)+
$$

\footnotetext{
${ }^{1}$ In this document we use the following definitions: $\gamma^{5}=i \gamma^{0} \gamma^{1} \gamma^{2} \gamma^{3}, \sigma_{\mu v}=\frac{i}{2}\left[\gamma_{\mu}, \gamma_{v}\right], \varepsilon^{\mu v \alpha \beta}$ is the totally antisymmetric fourth-rank tensor, $\varepsilon^{0123}=-1, e=\sqrt{4 \pi \alpha_{\mathrm{em}}}>0$, where $\alpha_{\mathrm{em}}$ is the electromagnetic coupling constant, $m_{b}$ is the $b$-quark mass, $m_{q}$ is the light $d$ or $s$ quark mass, $m_{Q}$ is the mass of $u$-or $c$-quark, $M_{1}$ is the mass of the initial $\bar{B}_{q}^{0}$ (or $B_{q}^{0}$ ) meson and $m$ is the mass of the charged lepton $\left(\ell^{+}\right.$or $\ell^{-}$).
} 


$$
\begin{aligned}
& +\frac{16}{3}\left(\lambda_{u}^{(q) *}+\lambda_{c}^{(q) *}\right) \frac{a_{1}}{C_{7 \gamma}} \frac{f_{B_{q}}}{m_{b}}, \\
F_{T A}^{\bar{b} \rightarrow \bar{q}}\left(q^{2}\right) & =\left(1-\frac{m_{q}}{m_{b}}\right)\left(F_{T A}\left(q^{2}, 0\right)+F_{T A}\left(0, q^{2}\right)\right) .
\end{aligned}
$$

Further, for construction of the matrix element of the rare radiative leptonic decyas we will use the dimensionless kinematical variables, normalized to the $B$-meson mass. That are the nondimetional masses $\hat{m}_{b}=m_{b} / M_{1}, \hat{m}_{q}=m_{q} / M_{1}, \hat{m}=m / M_{1}$, non-dimentional 4-momenta $\hat{p}=p / M_{1}$, $\hat{k}=k / M_{1}, \hat{k}_{1}=k_{1} / M_{1}, \hat{k}_{2}=k_{2} / M_{1}$ and non-dimensional Mandelstam variables:

$$
\hat{s}=(\hat{p}-\hat{k})^{2}, \quad \hat{t}=\left(\hat{p}-\hat{k}_{1}\right)^{2}, \quad \hat{u}=\left(\hat{p}-\hat{k}_{2}\right)^{2} .
$$

which satisfy the following relation $\hat{s}+\hat{t}+\hat{u}=1+2 \hat{m}^{2}$.

In the most common view the amplitude of the $\bar{B}_{q}^{0}\left(p, M_{1}\right) \rightarrow \gamma(k, \varepsilon) \ell^{+}\left(k_{1}\right) \ell^{-}\left(k_{2}\right)$ decay, neglecting bremstrahlung, may be written in the following way:

$$
\begin{gathered}
\left\langle\gamma(k, \varepsilon), \ell^{+}\left(k_{1}\right), \ell^{-}\left(k_{2}\right)\left|H_{\mathrm{eff}}^{\mathrm{SM} b \rightarrow q \ell^{+} \ell^{-}}\right| \bar{B}_{q}\left(p, M_{1}\right)\right\rangle=\frac{G_{F}}{\sqrt{2}} M_{1} \frac{\alpha_{e m}}{2 \pi} e V_{t b} V_{t q}^{*} \\
{\left[a(\mu, \hat{s}) \varepsilon_{\mu \varepsilon^{*} \hat{p} \hat{k}}\left(\bar{\ell}\left(k_{2}\right) \gamma^{\mu} \ell\left(-k_{1}\right)\right)-i b(\mu, \hat{s}) \varepsilon_{\mu}^{*}\left(\bar{\ell}\left(k_{2}\right) \gamma^{\mu} \ell\left(-k_{1}\right)\right)+\right.} \\
\left.e(\mu, \hat{s}) \varepsilon_{\mu \varepsilon^{*} \hat{p} \hat{k}}\left(\bar{\ell}\left(k_{2}\right) \gamma^{\mu} \gamma^{5} \ell\left(-k_{1}\right)\right)-i f(\mu, \hat{s}) \varepsilon_{\mu}^{*}\left(\bar{\ell}\left(k_{2}\right) \gamma^{\mu} \gamma^{5} \ell\left(-k_{1}\right)\right)\right] .
\end{gathered}
$$

For the Standard Model the $a(\mu, \hat{s})-f(\mu, \hat{s})$ functions are defined with the relations described in [3]. The amplitude of the $B_{q}^{0}\left(p, M_{1}\right) \rightarrow \gamma(k, \varepsilon) \ell^{+}\left(k_{1}\right) \ell^{-}\left(k_{2}\right)$ decay may be written in the following way:

$$
\begin{gathered}
\left\langle\gamma(k, \varepsilon), \ell^{+}\left(k_{1}\right), \ell^{-}\left(k_{2}\right)\left|H_{\mathrm{eff}}^{\mathrm{SM} b \rightarrow q \ell^{+} \ell^{-}}\right| B_{q}\left(p, M_{1}\right)\right\rangle=\frac{G_{F}}{\sqrt{2}} M_{1} \frac{\alpha_{e m}}{2 \pi} e V_{t b}^{*} V_{t q} \\
{\left[\tilde{a}(\mu, \hat{s}) \varepsilon_{\mu \varepsilon^{*} \hat{p} \hat{k}}\left(\bar{\ell}\left(k_{2}\right) \gamma^{\mu} \ell\left(-k_{1}\right)\right)+i \tilde{b}(\mu, \hat{s}) \varepsilon_{\mu}^{*}\left(\bar{\ell}\left(k_{2}\right) \gamma^{\mu} \ell\left(-k_{1}\right)\right)+\right.} \\
\left.e(\mu, \hat{s}) \varepsilon_{\mu \varepsilon^{*} \hat{p} \hat{k}}\left(\bar{\ell}\left(k_{2}\right) \gamma^{\mu} \gamma^{5} \ell\left(-k_{1}\right)\right)+i f(\mu, \hat{s}) \varepsilon_{\mu}^{*}\left(\bar{\ell}\left(k_{2}\right) \gamma^{\mu} \gamma^{5} \ell\left(-k_{1}\right)\right)\right] .
\end{gathered}
$$

All terms which make no contribution to the amplitudes in the $B_{q}$-meson rest frame are excluded from (1.6), (1.7). On transition from $\bar{B}_{q}^{0}$ to the $B_{q}^{0}$-mesons decays the $f(\mu, \hat{s})$ function changes it's sign, while $e(\mu, \hat{s})$ function doesn't. That may be explicitly seen in the definition of the amplitude (1.7). Functions $a(\mu, \hat{s})$ and $b(\mu, \hat{s})$ from (1.6) are replaced with new functions $\tilde{a}(\mu, \hat{s})$ and $\tilde{b}(\mu, \hat{s})$ which are also defined in [3]

The lepton bremsstrahlung contribution to the $\bar{B}_{q}^{0}\left(p, M_{1}\right) \rightarrow \gamma(k, \varepsilon) \ell^{+}\left(k_{1}\right) \ell^{-}\left(k_{2}\right)$ decay amplitude is described with the following expression [2]:

$$
\begin{aligned}
& \frac{G_{F}}{\sqrt{2}} M_{1} \frac{\alpha_{e m}}{2 \pi} e V_{t q}^{*} V_{t b} \frac{f_{B_{q}}}{M_{1}} \hat{m} C_{10 A} \times \\
& \times \bar{\ell}\left(k_{2}\right)\left[i\left(\frac{1}{\left(\hat{k}, \hat{k}_{2}\right)}-\frac{1}{\left(\hat{k}, \hat{k}_{1}\right)}\right)\left(\varepsilon^{*} \hat{p}\right)-\frac{i}{2}\left(\frac{1}{\left(\hat{k}, \hat{k}_{2}\right)}+\frac{1}{\left(\hat{k}, \hat{k}_{1}\right)}\right) \varepsilon_{\alpha \beta \mu \nu} \varepsilon^{* \alpha} \hat{p}^{\beta} \sigma^{\mu \nu}\right] \ell\left(-k_{1}\right) .
\end{aligned}
$$

The amplitude of the bremsstrahlung is $C$-invariant, it looks the same both for $\bar{B}_{q}^{0}$-and for $B_{q}^{0}$-decay (of course $V_{t q}^{*} V_{t b}$ is replaced by $V_{t q} V_{t b}^{*}$ ). This can be shown with the explicit calculations. 


\section{The EvtGen implementation of the $\bar{B}_{q}^{0}\left(B_{q}^{0}\right) \rightarrow \ell^{+} \ell^{-} \gamma$}

Recently the EvtGen package didn't include any model for the rare radiative leptonic decays of the $B$-mesons. Such a model, called BSTOGLLMNT, was introduced into the LHCb software framework as a part of the EvtGenExtras package since version v2r4. Practically it includes a set of the $\mathrm{C}++$ classes which implement the model and four EvtGen configuration files for the four decay channels, most interesting from the experimental point of view: $B_{d}^{0} \rightarrow \gamma \mu^{+} \mu^{-}, B_{d}^{0} \rightarrow \gamma e^{+} e^{-}$, $B_{s}^{0} \rightarrow \gamma \mu^{+} \mu^{-}$and $B_{s}^{0} \rightarrow \gamma e^{+} e^{-}$. However the generator is adopted for all three-body rare radiative leptonic decays which take place in the SM.

In addition the model contains the form-factors of the hadronic transitions, calculated within the dispersion formalizm of the quark model (with the the parametrizations adopted in [1, 8]) and the vector mesons dominance model [2]. It also includes the description of the dependence of the Wilson coefficients $C_{i}$ on the scale parameter $\mu$, the contribution from the vector resonances $\rho, \omega$, $\phi, J / \psi, \psi^{\prime}$ etc. within the Standard Model and different $C P$-violation effects according to [3].

The BSTOGLLMNT model is based on the following classes and methods:

- The Evtbs2ligammaMNT class serves as an interface between the BSTOGLLMNT model and the standard EvtGen classes.

- Evtbs2llGammaAmp class defines the amplitude and calculates the maximum probability for the rare radiative leptonic decays of the $B$-mesons. This class is also responsible for the application of a cut on the minimal photon energy and exclusion of the $J / \psi$ - and $\psi^{\prime}$ resonances region $(9.199<s<15.333)$. The latter option corresponds to the real experimental procedure of the search for the rare radiative leptonic decays.

- Class Evtbs2liGammaFFMNT calculates the form-factors of the $\bar{B}_{q}\left(B_{q}\right) \rightarrow \gamma$ decays, taking into account the $C P$-violation effects. For the numerical ansatz of the form-factors the expressions from the paper $[\overline{[}]$ are used.

- EvtbTosllWilsCoef fNLO class is responsible for calculation of the Wilson coefficients in the NLO approximation of the SM [ [\$]. This calculation takes into account the $C P$-violation effects [ [3] and (optionally) the contribution from the vector $u \bar{u}$ - and $c \bar{c}$-resonances. This class is taken from the BTOSLLMS model.

The hierarchy and the relations between different classes of the BSTOGLLMNT model is shown in Fig.11.

The generator provides a lot of freedom to the user. At the level of initial parameters input one may change the CKM-matrix elements, the scale parameter $\mu$, the number of the "effective" quark flavours used for the calculation of the strong interaction running constant $\alpha_{s}(\mu)$ and the minimal energy of the photon in the $B_{q}$ meson rest frame. It is also possible to define a choice of the $\alpha_{s}\left(M_{Z}\right)$ value (from lower to the upper limit of the experimental region).

\section{Monte-Carlo generator tests}

All the tests were performed for the case of $\mu^{+} \mu^{-}$-pair final state, as it is the closest to the real experiment needs. To check the model output some distributions for the generated particles have 


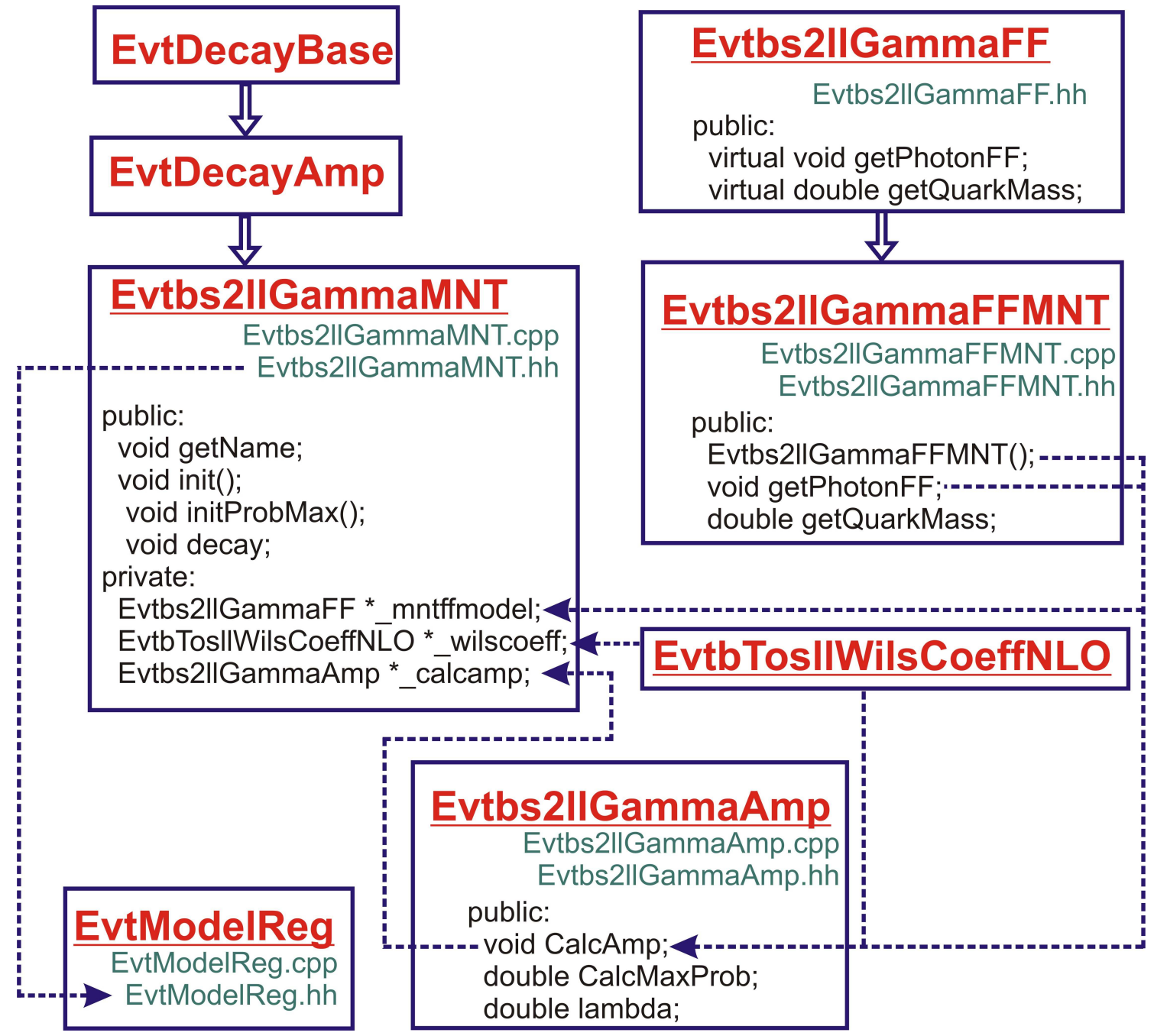

Figure 1: The hierarchy and relations of different classes in the BSTOGLLMNT model.

been plotted. First of them is the non-dimentional invariant mass of the lepton pair $\hat{s}$. As one of the tests it's useful to compare it with the theoretical prediction.

Dimuon invariant mass distribution for $B_{d}^{0}\left(\bar{B}_{d}^{0}\right) \rightarrow \gamma \mu^{+} \mu^{-}$decays is shown in Fig. 2 . Both plots are drawn in the double logarithmic scale. The left one presents the theoretical prediction and based on paper [2]. A solid line shows the distribution when all the resonance contributions are taken into account. A peak in the low $\hat{s}$ region corresponds to the $\omega$-meson. In the middle region the $J / \psi$ - and $\psi^{\prime}$-resonances may be observed. The photon pole and $\rho^{0}$-meson peak become too wide to be visible in the double logarithmic scale.

The right plot shows the output from the BSTOGLLMNT model with statistics of 30000 generated events. The photon pole, $\rho^{0}$ - and $\omega$-mesons contributions are contained in the first bin of the histogram. The region of $J / \psi$ - and $\psi^{\prime}$-resonances was excluded from the generation at the level of matrix element used in this model.

Fig. 3 shows the same distributions for the $B_{s}^{0}\left(\bar{B}_{s}^{0}\right) \rightarrow \gamma \mu^{+} \mu^{-}$decay. In the low $\hat{s}$ region the 

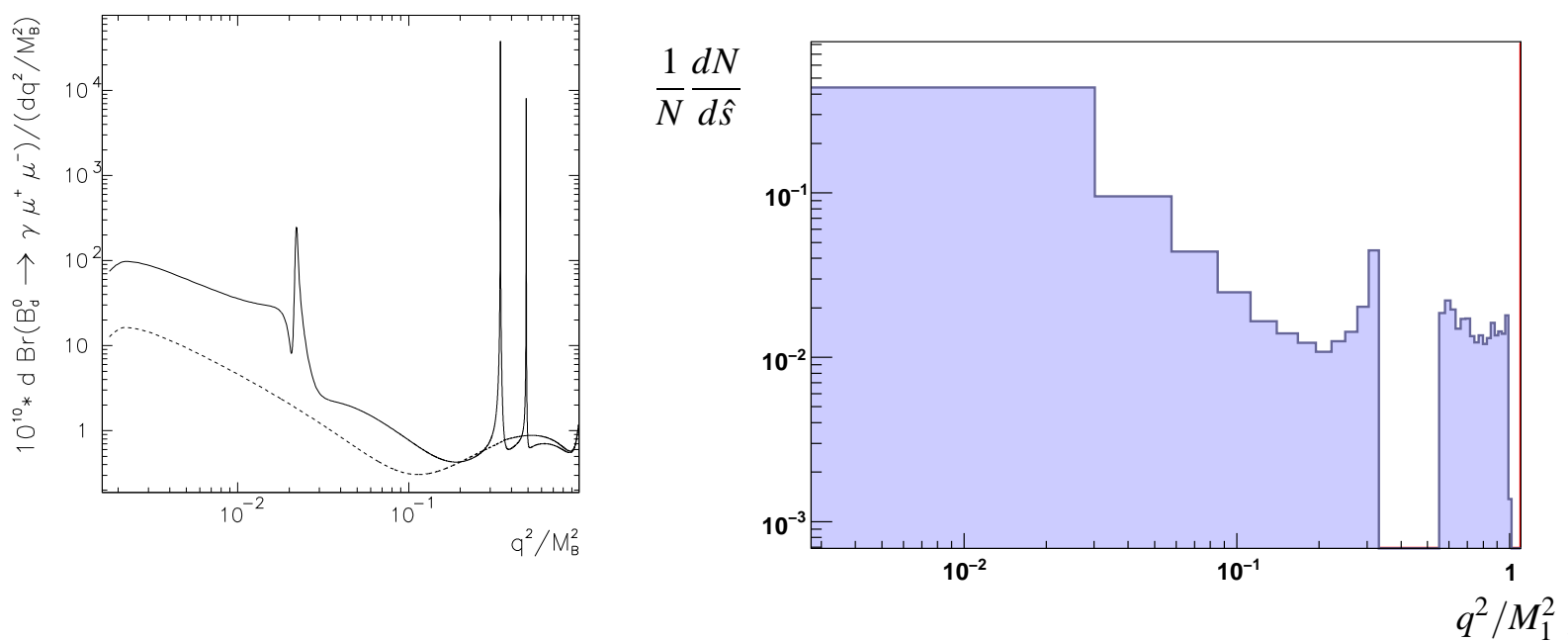

Figure 2: The non-dimentional invariant dimuon mass distribution for the $B_{d}^{0}\left(\bar{B}_{d}^{0}\right) \rightarrow \gamma \mu^{+} \mu^{-}$decay. The left plot shows the theoretical distribution from [2]. The right one is the normalized to unity distribution obtained in BSTOGLLMNT model. In these pictures $M_{1} \equiv M_{B}$.

$\phi$-meson peak dominates in the amplitude. For the Monte-Carlo distribution this peak contains about $90 \%$ of 36700 generated events.

Comparing plots in Fig.2 and 3 one may see a good agreement in the overall shape of the theoretical and Monte-Carlo distributions.

In addition, a test of the forward-backward asymmetry $A_{F B}(\hat{s})$ was performed. Its shape is very sensitive to the SM extentions, thus giving a very importrant information about the behaviour of the matrix element $d^{2} \Gamma / d \hat{s} d \cos \theta$. So, it is the most importrant differential distribution for the three-body decays of the $B$-mesons which have leptons in the final state.

In different papers there exist different definitions of the forward-backward asymmetries which may have oppisite signs. In the current note the following definition is used:

$$
A_{F B}(\hat{s})=\frac{\int_{0}^{1} d \cos \theta \frac{d^{2} \Gamma\left(\bar{B}_{q}^{0} \rightarrow \gamma \ell^{+} \ell^{-}\right)}{d \hat{s} d \cos \theta}-\int_{-1}^{0} d \cos \theta \frac{d^{2} \Gamma\left(\bar{B}_{q}^{0} \rightarrow \gamma \ell^{+} \ell^{-}\right)}{d \hat{s} d \cos \theta}}{\frac{d \Gamma\left(\bar{B}_{q}^{0} \rightarrow \gamma \ell^{+} \ell^{-}\right)}{d \hat{s}}}
$$

where $\theta$ is an angle between photon and negatively charged lepton momenta in the lepton pair rest frame. Neglecting the $C P$-violating effects and replacing the $\bar{B}_{q}$-meson with the $B_{q}$-meson in (3.1) will lead to the sign change to the opposite one.

In Fig. 4 one may see the theoretical asymmetry distribution (left) and the one generated within the BSTOGLLMNT model (right) for the $\bar{B}_{d}^{0} \rightarrow \gamma \mu^{+} \mu^{-}$decay. The gap in the middle $\hat{s}$ region corresponds to the excluded in the Monte-Carlo generator $J / \psi$ - and $\psi^{\prime}$-resonances.

Comparing the plots in Fig. 4 one may see a good agreement between the Monte-Carlo model results and theoretical predictions. 

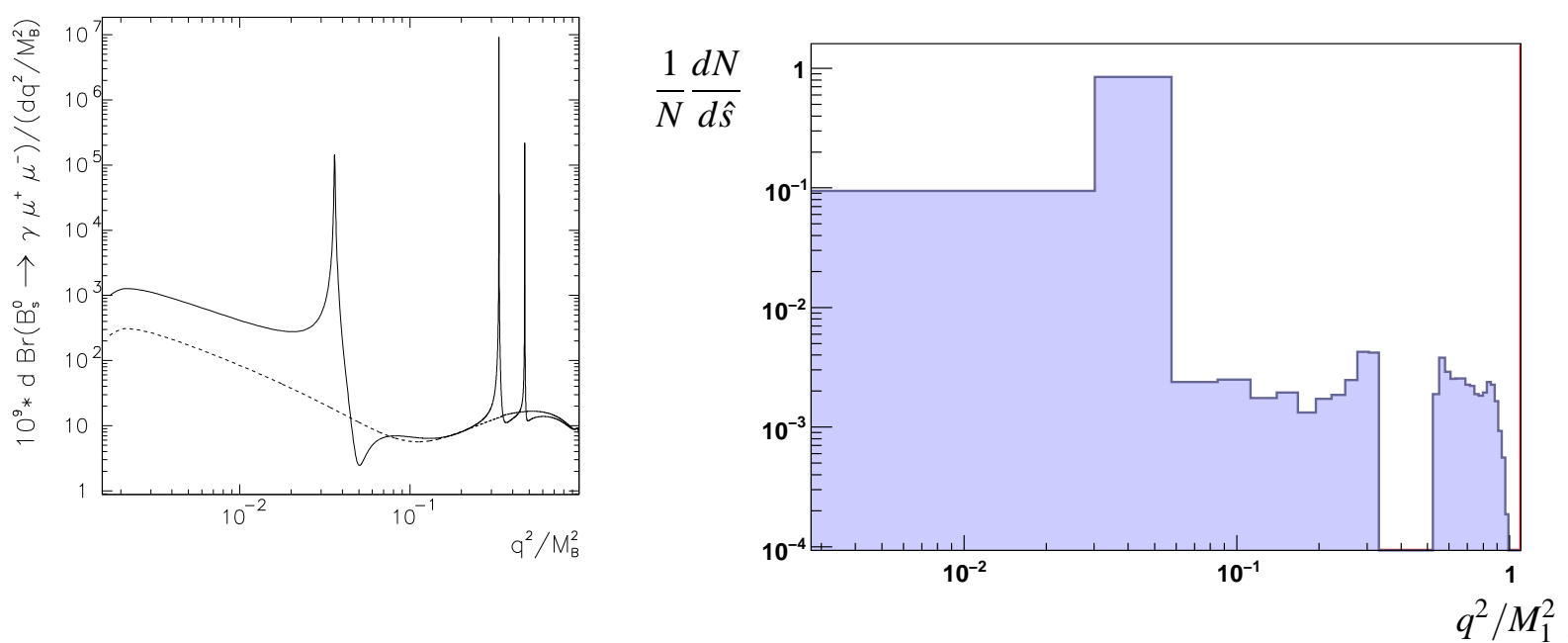

Figure 3: The non-dimentional invariant dimuon mass distribution for the $B_{s}^{0}\left(\bar{B}_{s}^{0}\right) \rightarrow \gamma \mu^{+} \mu^{-}$decay. The left plot shows the theoretical distribution from [2]. The right one is the normalized to unity distribution obtained in BSTOGLLMNT model. In these pictures $M_{1} \equiv M_{B}$.

Fig. 5 shows the forward-backward asymmetries for the $\bar{B}_{d}^{0} \rightarrow \gamma \mu^{+} \mu^{-}$(left) and $B_{d}^{0} \rightarrow \gamma \mu^{+} \mu^{-}$ (right) decays. The distributions are the mirror reflections of each other with an accuracy up to the number of events. The same result for the $\bar{B}_{s}^{0}$ and $B_{s}^{0}$ mesons is shown in Fig.6.

\section{Conclusion}

1) In the framework of the EvtGen package there was created a Monte-Carlo generator for the rare radiative leptonic decays $\bar{B}_{q}^{0}\left(B_{q}^{0}\right) \rightarrow \gamma \ell^{+} \ell^{-}$generation in the SM. The generator describes six different decay channels with $q=\{d, s\}$ and $\ell^{ \pm}=\left\{e^{ \pm}, \mu^{ \pm}, \tau^{ \pm}\right\}$.

2) The generator includes the form-factors of the hadronic transitions, calculated within the dispersion formalism of the quark model and the hypothesis of the domination of the vector mesons. The scale dependence of the Wilson coefficients and $C P$-violation effects are also included.

3) The generator output was tested with the statistics of about 30k events. It was shown, that neglecting the $C P$-violation effects, the result of the Monte-Carlo generation perfectly reproduces the theoretical predictions for the main differential distributions. The corresponding model, called BSTOGLLMNT, is included to the LHCb software framework.

\section{Acknowledgments}

The authors would like to thank their collegues Dr. D.Melikhov, Dr. K.Toms, Dr. G.Corti, Dr. W.Reece, a PhD student of the Physics Department of the Lomonosov Moscow State University I. Balakireva and an undergraduate student of the Physics Department of the Lomonosov Moscow State University I. Komarov for the fruitful discussions, their inspiration and great interest to this work. 

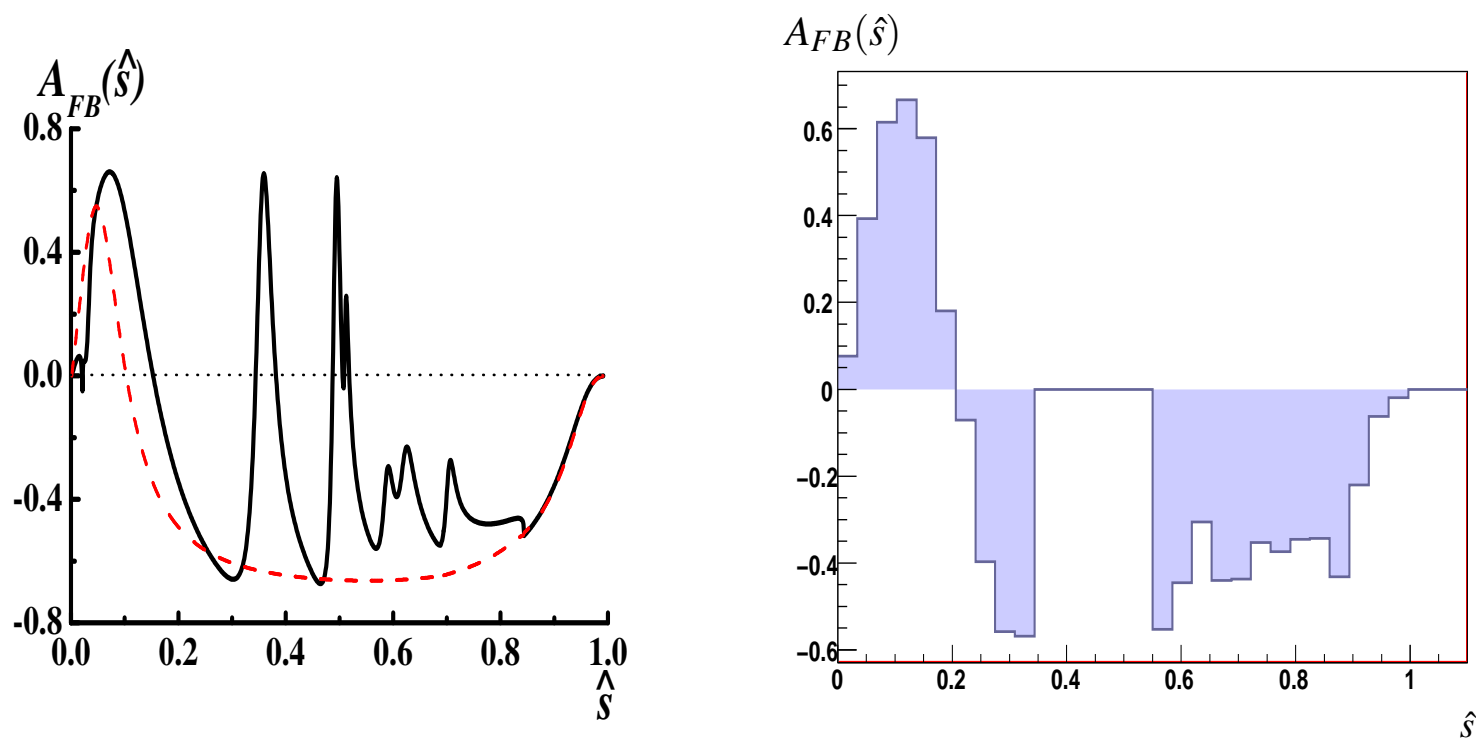

Figure 4: The forward-backward asymmetry $A_{F B}(\hat{s})$ for the $\bar{B}_{d}^{0} \rightarrow \gamma \mu^{+} \mu^{-}$decay. The left plot shows the theoretical prediction from []. The right plot show the Monte-Carlo distribution obtained within BSTOGLLMNT.

The job is partially supported by a grant from the President of the Russian Federation for Scientific Schools (4142.2010.2) and a grant from the Federal agency for science and innovations (02.740.11.0244) "The studies of the fundamental interactions of the elementary particles and the computing simulations for contemporary experiments".

\section{References}

[1] F. Kruger and D. Melikhov, Gauge invariance and form-factors for the decay $B \rightarrow \gamma \ell^{+} \ell^{-}$, Phys. Rev. D 67, 034002, (2003).

[2] D. Melikhov and N. Nikitin, Rare radiative leptonic decays $B_{d, s} \rightarrow \ell^{+} \ell^{-} \gamma$, Phys. Rev. D 70, 114028, (2004); D. Melikhov, N. Nikitin, and K. Toms, Rare radiative leptonic decays $B_{d, s} \rightarrow \ell^{+} \ell^{-} \gamma$, Phys. At. Nucl. 68, 1842 (2005).

[3] I. Balakireva, D. Melikhov, N. Nikitin and D. Tlisov, Forward-backward and CP-violating asymmetries in rare $B_{d, s} \rightarrow(\phi, \gamma) \ell^{+} \ell^{-}$decays, Phys. Rev. D 81, 054024, (2010).

[4] F. Kruger and L. M. Sehgal, $C P$ violation in the decay $B \rightarrow X(d) e^{+} e^{-}$, Phys. Rev. D 55, 2799 (1997); $C P$ violation in the exclusive decays $B \rightarrow \pi e^{+} e^{-}$and $B \rightarrow \rho e^{+} e^{-}, \mathbf{5 6}, 5452$ (1997); 60, 099905 Erratum (1999).

[5] T. Inami and C. S. Lim, Effects of Superheavy Quarks and Leptons in Low-Energy Weak Processes $K(L) \rightarrow \mu \bar{\mu}, K^{+} \rightarrow \pi^{+} v \bar{v}$ and $K^{0}-\bar{K}^{0}$, Prog. Theor. Phys. 65, 297 (1981); B. Grinstein, M. B. Wise, and M. J. Savage, $B \rightarrow X(s) e^{+} e^{-}$in the Six Quark Model, Nucl. Phys. B 319, 271 (1989); A. Buras and M. Munz, Effective Hamiltonian for $B \rightarrow X(s) e^{+} e^{-}$beyond leading logarithms in the NDR and HV schemes, Phys. Rev. D 52, 186 (1995); C. Bobeth et al., $\bar{B} \rightarrow X(s) \ell^{+} \ell^{-}$in the MSSM at NNLO, JHEP 0404, 071 (2004). 

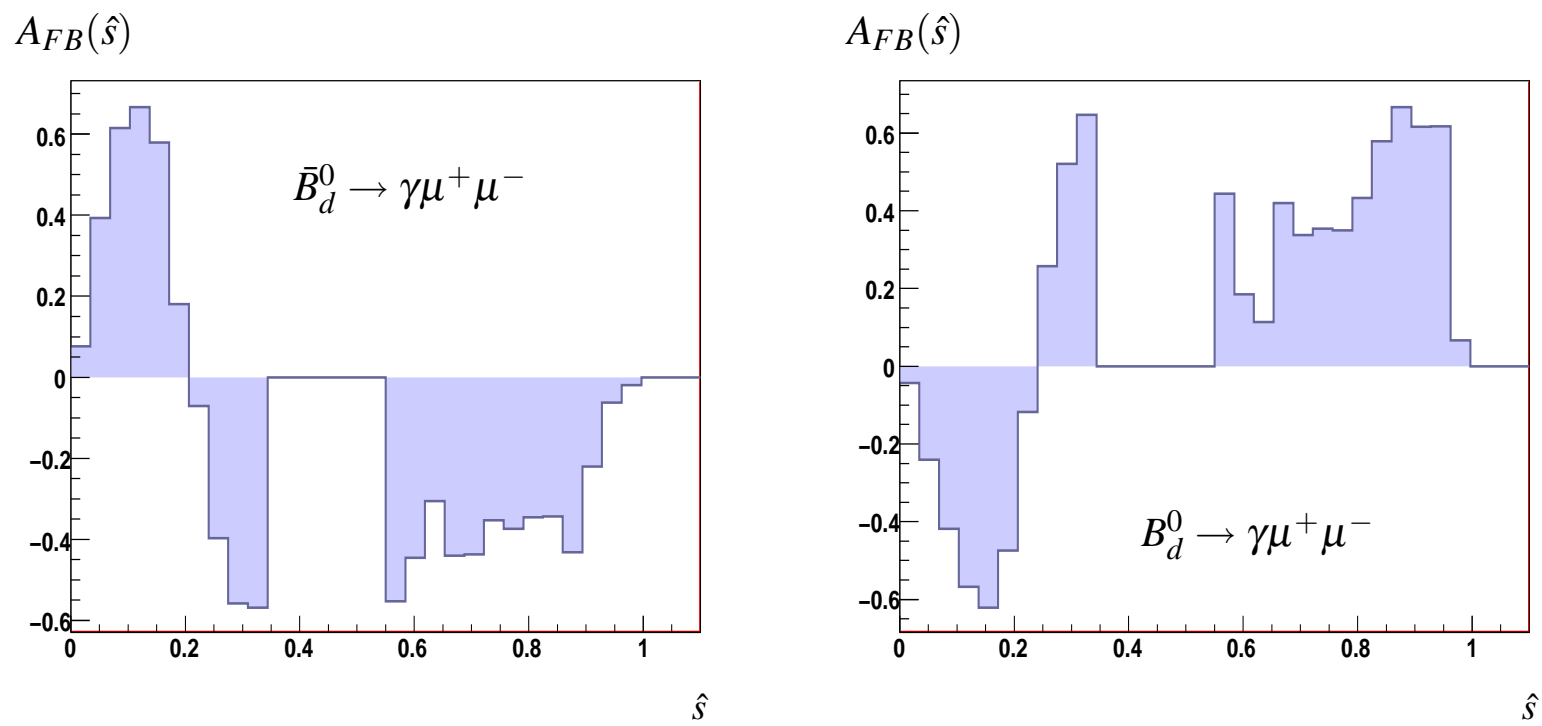

Figure 5: The forward-backward asymmetries $A_{F B}(\hat{s})$ for the $\bar{B}_{d}^{0} \rightarrow \gamma \mu^{+} \mu^{-}$(left) and $B_{d}^{0} \rightarrow \gamma \mu^{+} \mu^{-}$(right) decays, obtained within the BSTOGLLMNT.
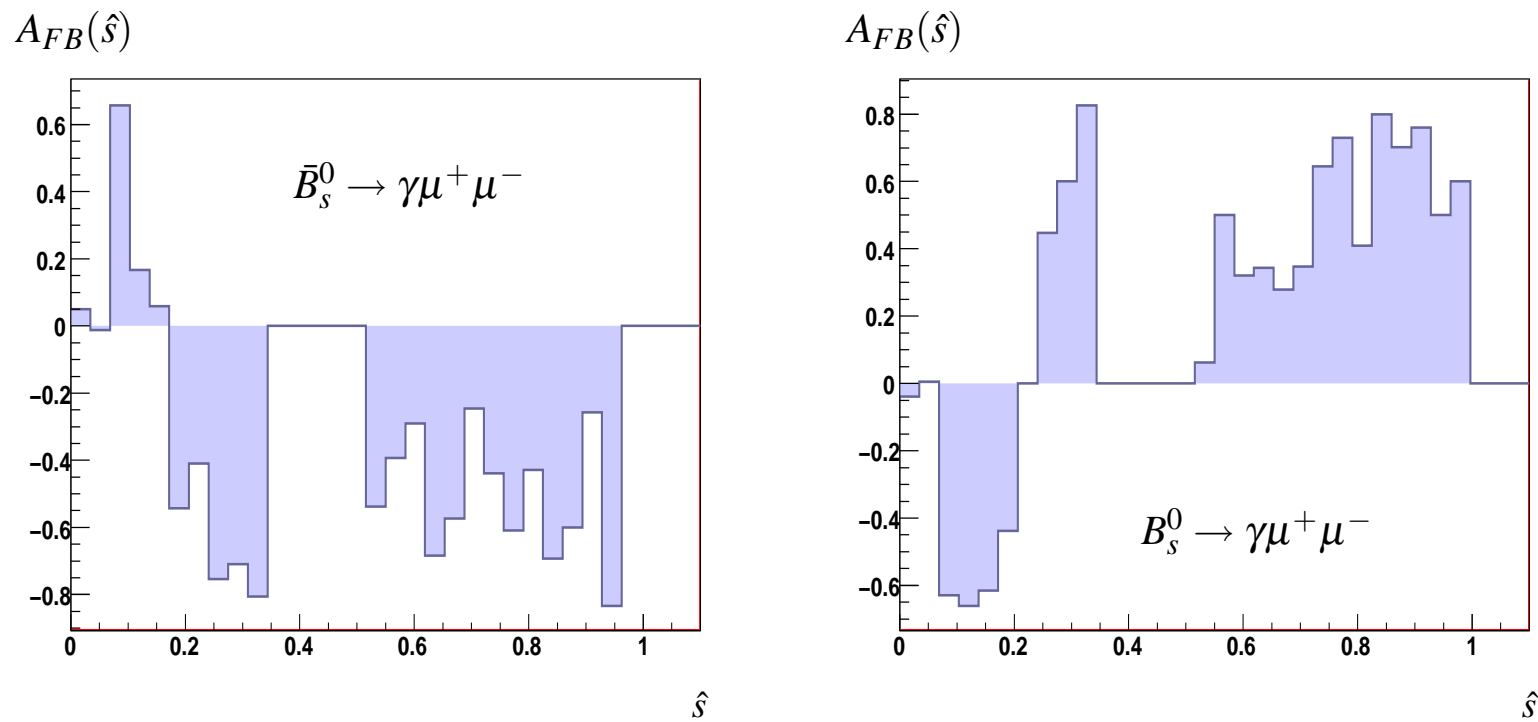

Figure 6: The forward-backward asymmetries $A_{F B}(\hat{s})$ for the $\bar{B}_{s}^{0} \rightarrow \gamma \mu^{+} \mu^{-}$(left) and $B_{s}^{0} \rightarrow \gamma \mu^{+} \mu^{-}$(right) decays, obtained within the BSTOGLLMNT.

[6] D. Melikhov, N. Nikitin, and S. Simula, Lepton asymmetries in exclusive $b \rightarrow s l^{+} l^{-}$decays as a test of the standard model, Phys. Lett. B 430, 332 (1998).

[7] D. Melikhov, O. Nachtmann, V. Nikonov, and T. Paulus, Masses and couplings of vector mesons from the pion electromagnetic, weak, and pi gamma transition form-factors, Eur. Phys. J. C 34, 345 (2004).

[8] D. Melikhov and B. Stech, Weak form-factors for heavy meson decays: An Update, Phys. Rev. D 62 , 014006 (2000).

[9] I. I. Bigi and A. I. Sanda, CP-Violation, 2 ed., (Cambridge University Press, 2009). 